\title{
Design, Development, and Testing of Flapping Fins with Actively Controlled Curvature for an Unmanned Underwater Vehicle
}

\author{
John Palmisano ${ }^{1}$, Jason Geder ${ }^{2}$, Ravi Ramamurti ${ }^{2}$, Kerr-Jia Liu ${ }^{3}$, Jonah \\ Cohen ${ }^{1}$, Tewodros Mengesha ${ }^{3}$, Jawad Naciri ${ }^{1}$, William Sandberg ${ }^{2}$, and Ba- \\ nahalli Ratna ${ }^{1}$ \\ ${ }^{1}$ Center for Biomolecular Science and Engineering, Naval Research Labo- \\ ratory, Washington, DC \\ ${ }^{2}$ Laboratory for Computational Physics and Fluid Dynamics, Naval Re- \\ search Laboratory, Washington, DC \\ ${ }^{3}$ Department of Mechanical and Aerospace Engineering, George Wash- \\ ington University, Washington, DC
}

Summary. This paper describes the design, construction, and testing of a biomimetic pectoral (side) fin with actively controlled curvature for UUV propulsion. It also describes the development of a test UUV and the design of a fin control system for vertical plane motion. A 3D unsteady computational fluid dynamics (CFD) analysis has been carried out to computationally optimize the fin design including a full study of the primary design parameters. The fin has been constructed and it can reproduce any specified deformation time-history. The full dynamics of the proposed vehicle have been modeled and the forces produced by the flapping fins computed. Finally, the stability of motion in the vertical plane has been analyzed and a control system has been designed.

Key words. Biomimetic pectoral fin, UUV, unsteady CFD, PID control, adaptive curvature

\section{Introduction}

Unmanned underwater vehicles (UUVs) have proven very useful in a wide range of applications including inspection, surveillance and exploration. Current technology allows UUVs to excel at complex operations from deep-sea diving to high-speed and long distance traversal. However, one weakness of this proven technology is low-speed, high maneuverabil- 
ity operations that are required for near-shore and littoral zone missions. A UUV designed for high performance in this niche area is essential to accomplish inspection and surveillance in such challenging environments.

To confront the issue of low-speed maneuverability in the presence of currents and near-shore obstacles, flapping fin mechanisms have been studied to understand how certain aquatic animals achieve their high levels of controllability. Blake (1979) determined that labriform locomotion, using pectoral fin oscillation with very little body undulation, is more efficient and allows for better maneuverability in low-speed operations than carangiform locomotion, using body and caudal fin oscillation. This result is important to UUV applications because artificial fins based on those of labriform swimmers can be mounted on a rigid hull without sacrificing internal volume for complex body actuation mechanisms. Kato (2000) developed a UUV based on the small-mouth bass with rigid flapping fins. Licht et al. (2004) presented a design for an underwater vehicle and investigated the issue of vehicle control, again using rigid flapping foils. Kato et al. (2002) and Ando et al. (2006) have developed both lift-based and drag-based deformable pectoral fins for use on UUVs. None of these fins, however, provide the precise control of surface curvature time-history that we have determined to be necessary to meet our performance goals for hovering and low-speed maneuvering.

Walker and Westneat (1997) experimentally studied fin kinematics of a class of lift-based labriform swimmers, specifically the bird wrasse, whose lift-based pectoral fin force production was a good match with our performance objectives. Ramamurti and Sandberg (2002) computationally studied force production by the bird wrasse and obtained good agreement with the results of Walker and Westneat (1997). We applied this validated computational method to the design and development of a biomimetic pectoral fin propulsor with actively controlled curvature.

We have chosen a two-fin test vehicle design in order to demonstrate deforming fin force production and vertical plane control in an underwater environment. We present a model of the dynamics of the vehicle for steady, level flight. The stability of motion in the vertical plane is analyzed, and vehicle geometries and linear control design techniques are considered.

\section{Fin Design}

The fin design begins with an in-depth analysis of the common bird wrasse pectoral fin, placing emphasis on fin kinematics, fluid dynamics, and anatomy. We identified dominant parameters and computationally in- 
vestigated their effects on force production. The results of our parametric study are presented to aid in future pectoral fin design. We then present the design and rationale of the physically constructed device that can produce the required fin kinematics. Lastly, the experimental measurements from our flapping fin tests are used to verify that the flapping fin is deforming in time as desired and to determine the actuator power required.

\subsection{CFD Parameterization}

To understand the effects of fin kinematics on propulsive force production, we used a 3D unsteady computational fluid dynamics code, FEFLO, which is an unstructured grid-based unsteady Navier-Stokes solver with automatic adaptive remeshing (Löhner 1988). Already successfully demonstrated by Ramamurti et al. (1996) and by Ramamurti and Sandberg (2001), this computational capability was extremely important in designing the pectoral fin. Not only did it help us rule out ineffective designs such as a rigid flapping fin (Ramamurti and Sandberg 2004), but also in testing design variations in search for performance gains. In this manner, we identified four major independent parameters that can be varied to change the force generation time-history during a stroke cycle: fin surface curvature, root angle of attack, bulk rotation angle, and frequency.

Fin surface curvature is defined as the shape of the fin with respect to time. There are 14 fin rays (also referred to as ribs) in the bird wrasse pectoral fin, each contributing to the fin curvature kinematics. For simplicity of design, manufacture, actuation and control, it is ideal to have the fewest possible number of ribs. But for more effective fin propulsion, it is ideal to maximize the number of ribs as more control points result in a smoother fit to desired fin curvature time-histories. Using CFD it was determined that 5 ribs resulted in a good balance between effective curvature and our specific mechanical implementation constraints (Ramamurti and Sandberg 2006). The overall rib and fin dimensions are scaled up from the bird wrasse pectoral fin to match our operational UUV requirements (Palmisano et al. (2007)). By imitating bird wrasse kinematics observed by Walker and Westneat (1997), we have determined an effective fin tip deflection time profile to define fin curvature. The curvature of each rib is defined by rib geometry and changes as a function of tip deflection. Due to a small deflection angle, rib 3 passively actuates between ribs 2 and 4 .

The angle of attack, $\alpha$, is the angle of the fin root with respect to the horizontal axis (Fig. 1). In the bird wrasse pectoral fin, this angle varies throughout the stroke cycle. However, a CFD analysis based on our simplified design and specific curvature time-history showed no thrust benefit 
to varying $\alpha$ over time. Instead, a fixed $\alpha$ of $20^{\circ}$, as seen in Fig. 1, was found to maximize forward thrust. This is consistent with the morphology of common labrid fish, as fast fish tend to have an $\alpha$ closer to $20^{\circ}$ while slow fish have an $\alpha$ closer to $70^{\circ}$ (Wainwright et al. 2002).

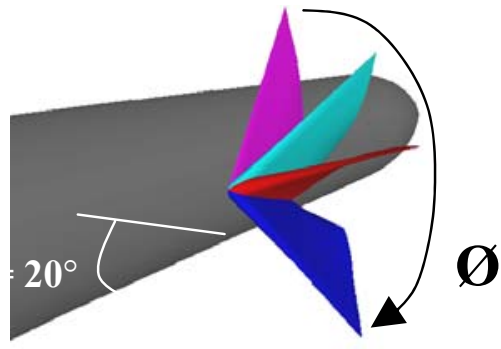

Fig. 1. $\alpha, \varnothing$, and fin curvature (at $1 \mathrm{~Hz}$ )

The bulk rotation angle, $\varnothing$, is the peak-to-peak amplitude of a stroke (Fig. 1). In the bird wrasse, this angle is about $74^{\circ}$. However, we found that as the bulk rotation angle increases, the thrust will also increase. We chose a nominal peak-to-peak amplitude of $114^{\circ}$ for our design, but this angle can be increased or decreased in flight by a controller for various operational conditions. An increase in bulk rotation angle also results in an increase of sinusoidal lift amplitude - an effect potentially degrading to UUV stability.

The fin flapping frequency was varied between $0.3 \mathrm{~Hz}$ and $3.3 \mathrm{~Hz}$ to study its effects on propulsion. It has been found that the upstroke thrust production increases as the square of the frequency ratio (Ramamurti and Sandberg 2006). A frequency of $1.2 \mathrm{~Hz}$ was chosen as a compromise between effective fin operation and mechanical limitations, although a higher frequency would be more desirable. Similar to varying the bulk rotation angle, changing the frequency can also be used as a vehicle control parameter.

\subsection{Construction}

Using the CFD results, and inspiration from the biological construction of the bird wrasse, we proceeded to construct the device. When encountering implementation conflicts, it was common to refer back to CFD to understand how a modification would affect propulsion. There are four main construction challenges: to design an actively deforming rib that we can exert full control over, to make a flexible skin connecting the ribs to serve 
as the fin surface area, to select small, lightweight, feedback controllable actuators that can independently bend each rib, and to devise a method for bulk rotation actuation.

Our fin design starts with the structural analysis, optimization, and construction of each of the five artificial ribs. Our fin rib is a compliant structure, designed to bend at specific base-to-tip deflection angles when subjected to translational forces at the base. The fin is composed of five of these independent ribs, and when actuated in particular patterns, their independent deflections are combined to create fin curvature as in Fig. 2.

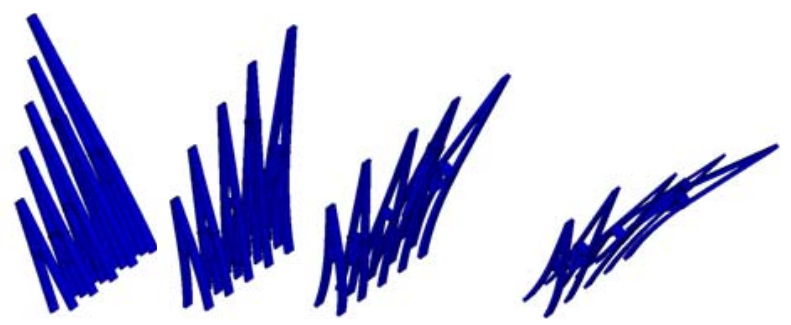

Fig. 2. Independently bending ribs forming pectoral fin curvature

The topology of the compliant ribs was designed by using a structuraloptimization based synthesis approach (Trease et al. 2003) and FEA with the ultimate objective to ensure the ribs could achieve the required deflection under fluid pressure when subjected to linear actuation at the base. The five designed ribs, each individually optimized based on different required lengths and curvatures, were built from ABS using a 3D printer. Details of the rib design are outlined by Palmisano et al. (2007).

A flexible skin of thickness $.4 \mathrm{~mm} \pm .1 \mathrm{~mm}$ is added to create a surface area between the individual ribs (Palmisano et al. 2007). This skin must easily stretch under rib deflection, yet not sag under fluid pressure. The skin was tested for chemical stability in sea water with no detectable degradation over a one month period. Skin thickness optimization was done experimentally and verified through FEA. Thick skin was shown to contribute significantly to required input force, while skin made too thin would tear. The empty space inside the fin is allowed to fill with water in order to equalize hydrostatic pressure and reduce skin sagging.

Servo motors are used as feedback controllable actuators for driving the ribs and bulk rotation (Fig. 3). Control in this manner is straightforward, simple to implement, and very affordable. All fin components were also made from $\mathrm{ABS}$ by $3 \mathrm{D}$ printing. 


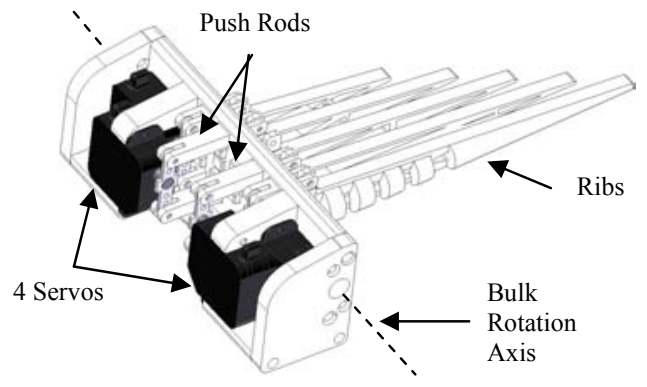

Fig. 3. Isometric view of fin system

All servos are controlled by the PIC16F877 microcontroller with a read-through list of pre-defined rotation angles and timing data points. Seven selected servo rotation angles for each of the four actuated ribs and the bulk motion are used during a complete stroke cycle. These angles are determined by manually measuring servo angle vs. rib curvature for each individual rib. The same microcontroller simultaneously collects all sensor data.

\subsection{Testing and Results}

Fin kinematics, generated forces, and power are measured from the fin. All measured data is for still water at a prescribed bulk rotation angle of $114^{\circ}$, an angle of attack of $0^{\circ}$, and a cycle frequency of $1.2 \mathrm{~Hz}$. Because testing occurs in still water, the $0^{\circ}$ angle of attack can be rotated in post processing calculations.
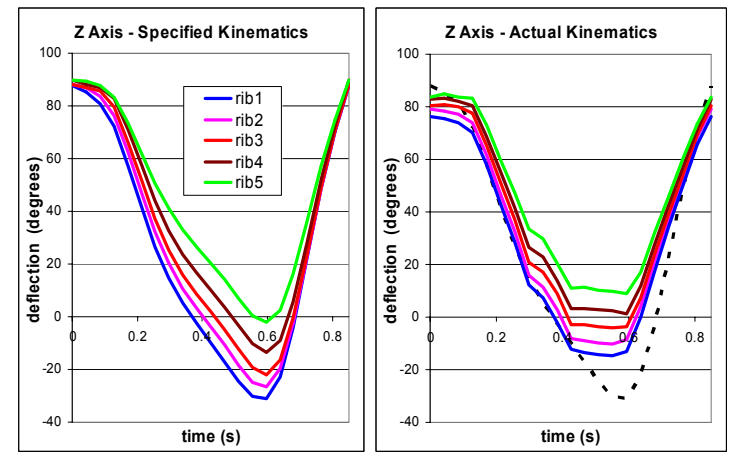

Fig. 4. (a) Specified CFD rib tip angle + bulk rotation angle $(1.2 \mathrm{~Hz})$. (b) Actual experimental camera measurements $(1.2 \mathrm{~Hz})$. 
To verify that the actual experimental kinematics matched the specified kinematics, we traced the 3D location of each rib tip across an entire cycle. Two high speed digital video cameras angled at $90^{\circ}$ to each other synchronously recorded images of the flapping fin at a predefined time step. Using image analysis software, matching rib tip coordinates selected from these image sequences were transformed into 3D coordinates (Geder and Sandberg 2007). Fig. 4 demonstrates good agreement between desired rib tip plus bulk rotation angles vs. actual experimental angles.

The measured thrust and lift shown in Fig. 5 are $0.028 \mathrm{~N}$ and $0.031 \mathrm{~N}$, respectively, while the CFD thrust and lift recomputed with experimental kinematics are $0.0262 \mathrm{~N}$ and $-0.0289 \mathrm{~N}$ (Palmisano et al. 2007). The expected differences in the force time histories between the experiment and CFD result from fluid-structure interaction and ingestion of air due to free surface interaction not present in CFD. Submerged vehicle testing will provide an environment more conducive to measuring the fin force production and propulsion efficiency.
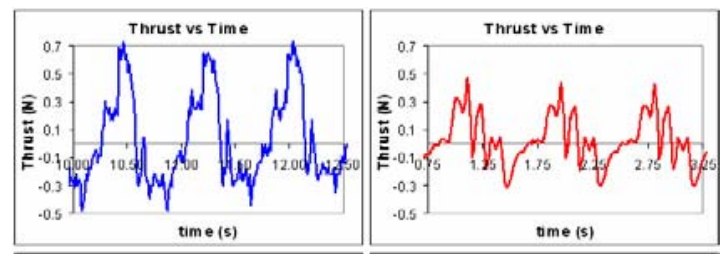

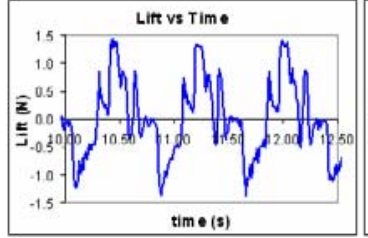

(a)

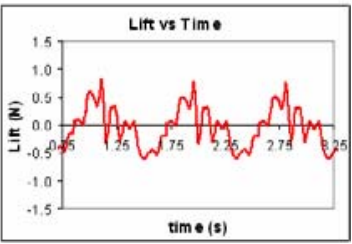

(b)

Fig. 5. (a) Measured forces over 3 cycles for experimental kinematics. (b) CFD computed forces over 3 cycles for experimental kinematics.

The total mean power consumption of all the servos was determined experimentally to be $9.81 \mathrm{~W}$, while control electronics consumed an additional 0.3W (Palmisano et al. 2007). This information was used for battery sizing, as described in the following section.

\section{Vehicle Design}

The goal of the test vehicle is to provide a platform which carries a set of two actively controlled curvature fins in order to demonstrate thrust 
production and vertical plane controllability in an underwater test environment. The flooded hull design measures 1.3" high, 13" long and 7" wide (Fig. 6). It has an out of water weight of $2.2 \mathrm{lb}$, and is slightly negatively buoyant in water. The center of mass is located near the center of buoyancy. Key hull design parameters were manufacturability, vertical plane stability, and drag reduction.

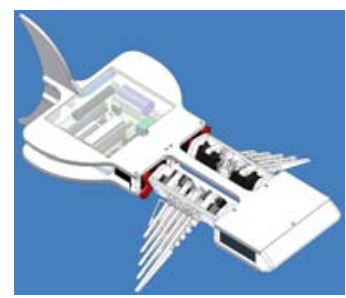

(a)

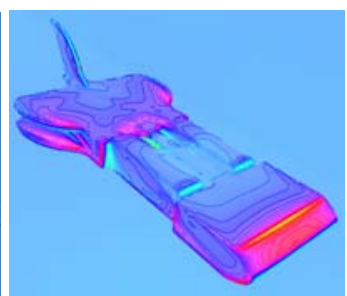

(b)

Fig. 6. (a) UUV design with two fins. (b) CFD drag analysis on UUV body

The horizontal flat design is not only easy to manufacture but also improves stability in the vertical plane. A vertical tail is incorporated in the design to provide stability in the horizontal plane.

The microcontroller has been designed to simultaneously control both fins, while making decisions based on mission goals and feedback from an accelerometer, a digital compass, and a depth sensor. The microcontroller has bootstrap software, allowing it to be reprogrammed in-vitro, as well as having wireless capabilities to transmit sensor data.

The prototype uses a $7.2 \mathrm{~V}$ NiMH $2600 \mathrm{mAh}$ battery with an experimentally calculated continuous full-speed runtime of 50 minutes. Actual expected battery life can be significantly longer, as the vehicle can go into various drift, position holding, and efficiency modes that consume much less energy.

\section{Vehicle Dynamics and Control System Design}

With the vehicle design finalized, we then modeled the dynamics of the system in order to investigate stability issues and determine the necessary control coefficients. For experimental verification of the modeled dynamics, we will quantitatively measure displacement, velocity, rotation, and acceleration in each plane by use of both on-board and off-board sensors. Propulsive forces can then be back-calculated using experimental data as input to a more sophisticated, transient trajectory computation (Alin et al. 2007). 


\subsection{Equations of Motion}

An undersea vehicle in motion is a complicated dynamic system. The vehicle has both rigid and elastic motions including control surface deflections and twisting of hydrodynamic surfaces. The elastic motion is observed in the bending and twisting of the controlled curvature fins. Isolating these fins, the rest of the body is treated as rigid, and as such, simplifications can be made by removing elastic terms from the equations of motion. We further reduce the equations by placing the body-fixed coordinate frame at the center of buoyancy and assuming symmetry about the $\mathrm{x}-\mathrm{z}$ plane.

\subsection{Evaluation of Forces and Moments}

The forces and moments acting on the vehicle can be represented by the sum of hydrodynamic, hydrostatic and pectoral fin forces and moments. Added mass is computed using Taylor series expansion and simplified geometry (Jakuba 2003). An exact solution of the hydrodynamic force and moment terms requires solving the Navier-Stokes equations with boundary conditions.

Because of the complex nature of the actively controlled curvature pectoral fins and the vorticity dynamics caused by the periodic flapping motion, 3-D unsteady CFD computations are needed to determine the force time-histories for an accurate UUV controls analysis. We derived all necessary force and moment coefficients and integrated them into the equations of motion, providing a fully described six degree-of-freedom system.

\subsection{Control System Design}

To verify the thrust and lift performance of the fins, straight line motion of a vehicle is first considered. In this mode of motion, sway, yaw and roll $(Y, N, L)$ can be assumed zero leaving us with 3DOF motion in the vertical plane characterized by surge, heave and pitch $(X, Z, M)$. A linearized set of vertical plane equations (Eq. 4.1) about steady, level flight allows use of classical control techniques and provides an adequate representation of the system in the neighborhood of this flight condition (Fig. 7c). Steady level flight is characterized by a nominal surge velocity and by zero values for heave and pitch velocities, and pitch angle. Similar linearizations are carried out for other maneuvers including climbing and diving. Later control systems will incorporate nonlinear control techniques as the need for maneuverability across all flight conditions arises. 


$$
\begin{aligned}
& m\left(\dot{u}+z_{G} \dot{q}\right)= \\
& \quad X_{u|u|} u_{0}|u|+X_{\dot{u}} \dot{u}+X_{u w} u_{0}|w|-(W-B) \theta+X_{p e c} \\
& m\left(\dot{w}-u_{0} q+x_{G} \dot{q}\right)= \\
& \quad Z_{u w} u_{0} w+Z_{u q} u_{0} q+Z_{\dot{w}} \dot{w}+Z_{\dot{q}} \dot{q}+(W-B)+Z_{p e c} \\
& I_{y} \dot{q}-m x_{G}\left(\dot{w}-u_{0} q\right)+m z G^{\dot{u}}= \\
& \quad M_{u w} u^{w}+M_{u q} u_{0} q+M_{\dot{w}} \dot{w}+M_{\dot{q}} \dot{q}-W z_{G} \theta-W x_{G}+M_{p e c}
\end{aligned}
$$

Representing the system from Eq. 4.1 in matrix form allows us to determine stability by implementing an open-loop Bode analysis. Fig. 7a shows that the system is stable in pitch and heave velocities without any form of closed loop control. However, to navigate to specified vehicle waypoints properly, feedback control is necessary.

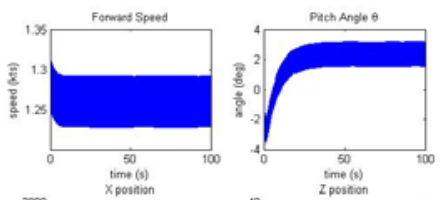

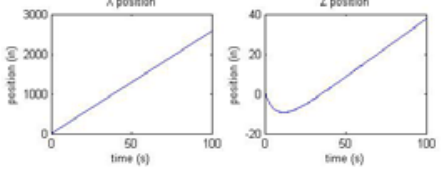

(a)

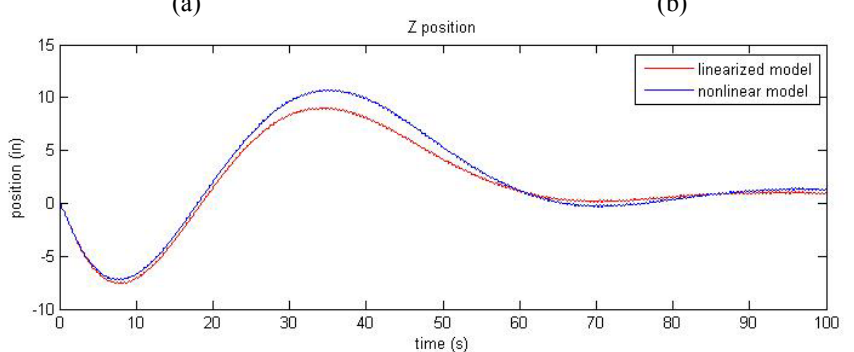

(c)
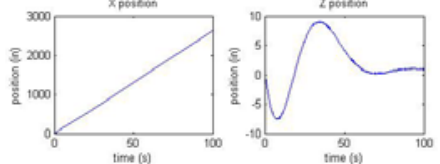

(b)
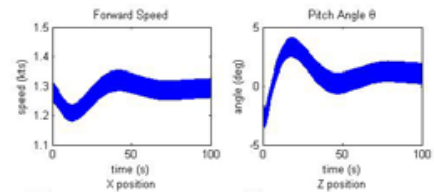

Fig. 7. (a) Open-loop vehicle response to fin operating at $2 \mathrm{~Hz}$. (b) Closed-loop vehicle response to control of depth by way of fin frequency. (c) Closed-loop vehicle response comparison between a full nonlinear model of the system and a model linearized about steady, level flight.

Adding feedback control to the system, as shown in Fig. 8, provides the means for the UUV to respond to commands or a changing environment within specified performance parameters. Proportional-integralderivative (PID) control has been implemented as a simple and effective means of controlling our vehicle for the desired testing. When control over a wider range of operating conditions is required in future testing, more robust, nonlinear control schemes will be implemented. Controllable 
parameters for the vehicle include pectoral fin flapping frequency and stroke amplitude. Altering these variables produces an increase or decrease in thrust and lift.

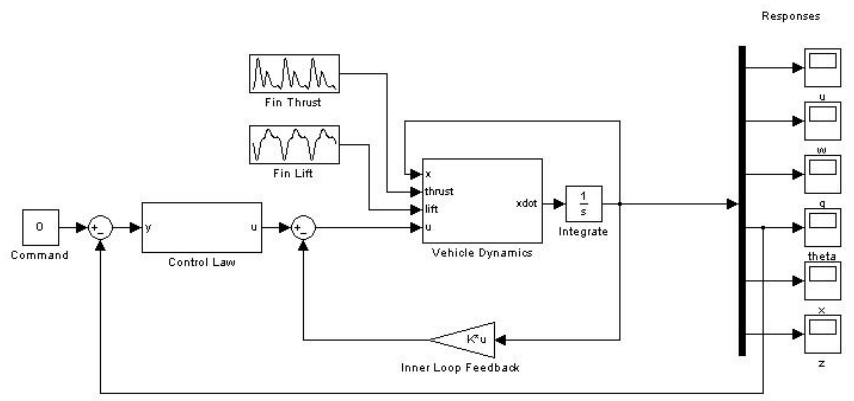

Fig. 8. Block diagram of vehicle feedback control system

As an example, the responses in Fig. $7 \mathrm{~b}$ are a result of using depth as the feedback control variable with fin flapping frequency as the control input. In this simulation, we have discretized the control input to only change every 3 seconds. This allows the fins to operate at a single frequency over 4-5 cycles preserving the integrity of each stroke. With PID control, we have successfully stabilized depth at a commanded value.

\section{Conclusions}

We have described the design, development, and testing of actively controlled curvature pectoral fins for use on a UUV and demonstrated the achievement of a desired curvature time-history. We also have described the prototype vehicle that has been designed to incorporate these fins. The 3-D unsteady CFD analysis used to assist in the fin design and development processes, as well as for the vertical plane controller design, has also been described.

\section{References}

Alin N, Fureby C, Svennberg SU, Sandberg WC, Ramamurti R, Bensow RE (2007) Large Eddy Simulation of the Transient Flow Around A Submarine During A Maneuver. $45^{\text {th }}$ AIAA Aerospace Sciences Meeting and Exhibit, AIAA-2007-1454, Reno, NV

Ando Y, Kato N, Suzuki H, Ariyoshi T, Suzumori K, Kanda T, Endo S (2006) Elastic Pectoral Fin Actuators for Biomimetic Underwater Vehicles. Proc of the $16^{\text {th }}$ International Offshore and Polar Engineering Conference, pp 260-267 
Blake RW (1979) The mechanics of labriform motion I. Labriform locomotion in the angelfish (pterophyllum eimekei): An analysis of the power stroke. J Exp Biol 82:255-271

Geder J, Sandberg WC (2007) Multi-Camera, High-Speed Imaging System for Flapping Fin Kinematics Measurement. Naval Research Laboratory Memorandum Report NRL/MR 6401-07. Submitted for publication January 2007

Jakuba M (2003) Modeling and Control of an Autonomous Underwater Vehicle with Combined Foil/Thruster Actuators. SM Thesis, WHOI-MIT Joint Program in Oceanographic Engineering

Kato N (2000) Control performance in horizontal plane of fish robot with mechanical pectoral fins. IEEE J Ocean Eng 25:121-129

Kato N, Liu H, Morikawa H (2002) Biology-Inspired Precision Maneuvering of Underwater Vehicles. Proc of the $12^{\text {th }}$ International Offshore and Polar Engineering Conference, vol 2, pp 269-276

Licht S, Polidoro V, Flores M, Hover FS, Triantafyllou MS (2004) Design and Projected Performance of a Flapping Foil AUV. IEEE J Ocean Eng 29:786794

Löhner R (1988) An adaptive finite element solver for transient problems with moving bodies. Computers \& Structures 30:303-317

Palmisano J, Ramamurti R, Lu K, Cohen J, Sandberg W, Ratna B (2007) Design of a Biomimetic Controlled-Curvature Robotic Pectoral Fin. IEEE International Conference on Robotics and Automation, Rome, IT

Ramamurti R, Sandberg WC (2001) Simulation of Flow About Flapping Airfoils Using A Finite Element Incompressible Flow Solver, AIAA J., 39 (2):253-260

Ramamurti R, Sandberg WC (2002) Fluid dynamics of flapping aquatic flight in the bird wrasse: three-dimensional unsteady computations with fin deformation. J Exp Biol 205:2997-3008

Ramamurti R, Sandberg WC (2004) The influence of fin rigidity and gusts on force production in fishes and insects: a computational study, $42^{\text {nd }}$ AIAA Aerospace Sciences Meeting, AIAA 2004-404, Reno, NV

Ramamurti R, Sandberg WC (2006) Computational Fluid Dynamics Study for Optimization of a Fin Design. $24^{\text {th }}$ AIAA Applied Aerodynamics Conference, AIAA-2006-3658, San Francisco, CA

Ramamurti R, Löhner R, Sandberg WC (1996) Computation of unsteady flow past a tuna with caudal fin oscillation. In: Rahman M, Brebbia CA (Eds) Advances in Fluid Mechanics Vol. 9. Computational Mechanics Publications, Southampton, UK, pp 169-178

Trease BP, Lu KJ, Kota S (2003) Biomimetic Compliant System for Smart Actuator-Driven Aquatic Propulsion: Preliminary Results. ASME Int. Mechanical Eng. Congress \& Exposition, IMECE2003-41446

Wainwright P, Bellwood D, Westneat M (2002) Ecomorphology of Locomotion in Labrid Fishes. Environmental Biology of Fishes 65:47-62

Walker JA, Westneat MW (1997) Labriform Propulsion in Fishes: Kinematics of Flapping Aquatic Flight in the Bird Wrasse, Gomphosus Varius (Labridae). J Exp Biol 200:1549-1569 